PROCEEDINGS OF THE

AMERICAN MATHEMATICAL SOCIETY

Volume 125, Number 2, February 1997, Pages 611-615

S 0002-9939(97)03871-9

\title{
ON A CLASS OF SUBALGEBRAS OF $C(X)$ AND THE INTERSECTION OF THEIR FREE MAXIMAL IDEALS
}

\author{
S. K. ACHARYYA, K. C. CHATTOPADHYAY, AND D. P. GHOSH
}

(Communicated by Franklin D. Tall)

\begin{abstract}
Let $X$ be a Tychonoff space and $A$ a subalgebra of $C(X)$ containing $C^{*}(X)$. Suppose that $C_{K}(X)$ is the set of all functions in $C(X)$ with compact support. Kohls has shown that $C_{K}(X)$ is precisely the intersection of all the free ideals in $C(X)$ or in $C^{*}(X)$. In this paper we have proved the validity of this result for the algebra $A$. Gillman and Jerison have proved that for a realcompact space $X, C_{K}(X)$ is the intersection of all the free maximal ideals in $C(X)$. In this paper we have proved that this result does not hold for the algebra $A$, in general. However we have furnished a characterisation of the elements that belong to all the free maximal ideals in $A$. The paper terminates by showing that for any realcompact space $X$, there exists in some sense a minimal algebra $A_{m}$ for which $X$ becomes $A_{m}$-compact. This answers a question raised by Redlin and Watson in 1987. But it is still unsettled whether such a minimal algebra exists with respect to set inclusion.
\end{abstract}

\section{INTRODUCTION}

One of the fascinating problems considered in Gillman and Jerison [2] is that of characterising the intersection of all the free maximal ideals in the algebra $C(X)$ of real-valued continuous functions on a Tychonoff space $X$ and its subalgebra $C^{*}(X)$ of bounded functions. Suppose $C_{K}(X)$ is the set of all functions in $C(X)$ which have compact support, and let $C_{\infty}(X)$ consist of exactly those functions $f$ in $C(X)$ which vanish at $\infty$ in the sense that $\left\{x \in X:|f(x)| \geq \frac{1}{n}\right\}$ is compact for each $n$ in $\mathbb{N}$. Kohls [3] has shown that the intersection of all the free ideals in $C(X)$ or in $C^{*}(X)$ is $C_{K}(X)$. We have established the truth of the same result for a subalgebra $A$ of $C(X)$ that contains $C^{*}(X)$. Kohls [3] has further proved that the intersection of all the free maximal ideals in $C^{*}(X)$ is precisely the set $C_{\infty}(X)$. Incidentally it is shown in [2] that for a realcompact space $X, C_{K}(X)$ is identical to the intersection of all the free maximal ideals in $C(X)$. In this paper we show that for a subalgebra $A$ of $C(X)$ containing $C^{*}(X)$, each element $f$ belonging to the intersection of all the free maximal ideals in $A$ is characterised by the property that $\left\{x \in X:|f(x) g(x)| \geq \frac{1}{n}\right\}$ is compact for each $n$ in $\mathbb{N}$ and for each $g$ in $A$. It is interesting to note that this result puts the two earlier results into a common setting.

Received by the editors February 11, 1994 and, in revised form, January 30, 1995.

1991 Mathematics Subject Classification. Primary 54C40; Secondary 46E25.

Key words and phrases. Algebra of continuous functions, maximal ideal, compactification, realcompactification. 
Redlin and Watson [5] introduced the notion of $A$-compactness of which compactness and realcompactness are particular cases. According to this terminology a compact space is $C^{*}$-compact while a realcompact space is $C$-compact. In view of the result of the last paragraph, note that if $A=C(X)$ or $C^{*}(X)$ and $X$ is $A$ compact, then $C_{K}(X)$ is identical to the intersection of all the free maximal ideals in $A$. We have constructed an example which shows that such a conclusion is not true in general for an arbitrary $A$-compact space.

We conclude the paper by showing that given any realcompact space $X$, there exists in some sense a minimal algebra $A_{m}$ lying between $C(X)$ and $C^{*}(X)$ for which $X$ becomes $A_{m}$-compact. This gives an answer to the question raised by Redlin and Watson [5]. It has further been shown that a minimal algebra thus obtained need not be minimal with respect to set inclusion, however it still remains open whether such a minimal algebra exists with respect to set inclusion.

\section{INTERSECTION OF FREE MAXIMAL IDEALS}

Throughout the paper $X$ stands for a Tychonoff space and subalgebras of $C(X)$ are supposed to contain $C^{*}(X)$. For any $f$ in $C(X), Z(f)$ will denote the zero-set $\{x \in X: f(x)=0\}$. Ideals of subalgebras of $C(X)$ are assumed to be proper. An ideal $I$ in a subalgebra $A$ of $C(X)$ is called fixed if $\bigcap Z[I] \neq \emptyset$, otherwise $I$ is said to be free. Each member $f$ of $C(X)$ has a unique continuous extension $f^{*}: \beta X \longrightarrow \mathbb{R}^{*}$, where $\mathbb{R}^{*}$ is the one-point compactification of $\mathbb{R}$; if $f \in C^{*}(X)$, $f^{*}$ is the same as $f^{\beta}$, the unique extension of $f$ to $\beta X$. Plank [4] has shown that the family of all the maximal ideals in $A$ is precisely the set $\left\{M_{A}^{p}: p \in \beta X\right\}$ where $M_{A}^{p}=\left\{f \in A:(f g)^{*}(p)=0 \forall g \in A\right\}$; and $M_{A}^{p}$ is a free ideal if and only if $p$ belongs to $\beta X-X$.

If $F$ is the intersection of all the free ideals in $A$, then it is easy to show that $C_{K}(X) \subset F$. On the other hand, $C_{K}(X)=\bigcap_{p \in \beta X-X} O^{p}$ (see [2], 7E) where for each $p$ in $\beta X, O^{p}=\left\{f \in C(X): c_{\beta X} Z(f)\right.$ is a neighbourhood of $\left.p\right\}$. Furthermore for each $p$ in $\beta X, O^{p}$ is the intersection of all the prime ideals containing it and contained in $M_{C}^{p}$ and hence

$$
C_{K}(X)=\bigcap_{p \in \beta X-X}\left\{P \cap A: O^{p} \subset P \subset M_{C}^{p}, P \text { is a prime ideal in } C(X)\right\} .
$$

It is clear that for any prime ideal $P$ in $C(X)$ appearing on the right side of the above equality, $P \cap A$ is a free prime ideal of $A$. Hence $F \subset C_{K}(X)$. Thus we have the following result.

Theorem 2.1. $C_{K}(X)$ is the intersection of all the free ideals in $A$.

In order to describe the intersection of all the free maximal ideals in $A$, let $A_{\infty}(X)$ denote the family of all functions $f$ in $A$ for which the set $A_{n}(f g)=$ $\left\{x \in X:|f(x) g(x)| \geq \frac{1}{n}\right\}$ is compact for each $n$ in $\mathbb{N}$ and each $g$ in $A$. If $f$ belongs to $A_{\infty}(X), g$ is in $A, p$ belongs to $\beta X-X$ and $\epsilon>0$, then it is easy to see in view of the continuity of $(f g)^{\beta}$ at $p$ and denseness of $X$ in $\beta X$ that $\left|(f g)^{\beta}(p)\right|<\frac{1}{n}+\epsilon$ for each $n$ in $\mathbb{N}$. Consequently $\beta X-X \subset Z\left((f g)^{\beta}\right)$ and hence $A_{\infty}(X) \subset \bigcap\left\{M_{A}^{p}: p \in \beta X-X\right\}$. Conversely, if $f$ belongs to $M_{A}^{p}$ for each $p$ in $\beta X-X$ and $g$ is in $A$, then $f g$ belongs to $C^{*}(X)$ and $\beta X-X \subset Z\left((f g)^{\beta}\right)$. We claim that $A_{n}(f g)$ is compact. If not, then there exists $p$ in $c l_{\beta X} A_{n}(f g)-A_{n}(f g)$ for which $(f g)^{\beta}(p)=0$. But $|f g|^{\beta}\left(c l_{\beta X} A_{n}(f . g)\right) \subset c l_{\mathbb{R}}\left(|f . g|\left(A_{n}(f . g)\right)\right) \subset\left[\frac{1}{n}, \infty\right)$ - a contradiction. Therefore we have the following result: 
Theorem 2.2. $A_{\infty}(X)$ is the intersection of all the free maximal ideals in $A$.

We note that if $X$ is realcompact and $A=C(X)$, then $A_{\infty}(X)$ is the family of all functions in $C(X)$ with compact support, and so Theorem 8.19 of [2] follows from our Theorem 2.2. On the other hand if $A=C(X)$, then $A_{\infty}(X)$ and $C_{\infty}(X)$ are identical and hence Lemma 3.2 of [3] is also a special case of Theorem 2.2 .

\section{A-COMpaCtness}

Following Redlin and Watson [5], we define a maximal ideal $M$ in $A$ to be real if the quotient field $A / M$ is isomorphic to $\mathbb{R}$, otherwise $M$ is called hyperreal. $X$ is called $A$-compact if every real maximal ideal in $A$ is fixed. In view of this definition it follows that a compact space is $C^{*}$-compact while a realcompact space is $C$ compact.

As in [2], 7.9(b), one can prove the following lemma.

Lemma 3.1. For each $p$ in $\beta X, M_{A}^{p}$ is hyperreal if and only if $M_{C^{*}}^{p}$ contains a unit of $A$.

In what follows we give a useful characterisation of $A$-compactness.

Theorem 3.2. A space $X$ is $A$-compact if and only if for every $p$ in $\beta X-X$, there exists an $f$ in $C^{*}(X)$ such that $f$ is a unit of $A$ and $f^{\beta}(p)=0$ (or equivalently $X$ is $A$-compact if and only if for every $p$ in $\beta X-X$, there exists a unit $g$ of $A$ such that $g^{-1} \in C^{*}(X)$ and $\left.g^{*}(p)=\infty\right)$.

Proof. Let $X$ be $A$-compact and $p \in \beta X-X$. Then $M_{A}^{p}$ is hyperreal and hence by Lemma 3.1, $M_{C^{*}}^{p}=\left\{h \in C^{*}(X): h^{\beta}(p)=0\right\}$ contains a unit $f$ of $A$. Clearly $f^{\beta}(p)=0$. Conversely, let the given condition hold. Then for any $p \in \beta X-X$, $f^{\beta}(p)=0$ for some $f$ in $C^{*}(X)$ with $f$ a unit of $A$. Since now $f \in M_{C^{*}}^{p}$, Lemma 3.1 implies that $M_{A}^{p}$ is hyperreal and hence $X$ is $A$-compact.

Remark 3.3. If we take $A=C(X)$ in the above theorem, then we have the following result.

$X$ is realcompact if and only if each point of $\beta X-X$ is contained in a zero-set in $\beta X$ which misses $X$.

This is in fact the content of a theorem of Hewitt (see [6], page 31).

Note that if $X$ is $C$-compact (respectively $C^{*}$-compact), then $C_{K}(X)$ is the same as the intersection of all the free maximal ideals in $C(X)$ (respectively $C^{*}(X)$ ). The following example shows that this is not true for an arbitrary $A$-compact space. In what follows for any subfamily $\mathcal{F}$ of $C(X)$, the subset $\mathcal{A}(\mathcal{F})$ will stand for the smallest subalgebra of $C(X)$ containing $\mathcal{F}$.

Example 3.4. Consider $A=\mathcal{A}\left(C^{*}(\mathbb{N}) \cup\{i\}\right)$, where $i(n)=n$ for each $n$ in $\mathbb{N}$. Since $Z\left(j^{\beta}\right)=\beta \mathbb{N}-\mathbb{N}$, where $j=i^{-1}$, it follows from Theorem 3.2 that $\mathbb{N}$ is $A$ compact. Let $h$ in $C^{*}(\mathbb{N})$ be defined as $h(n)=e^{-n}$ for each $n$ in $\mathbb{N}$. Then $h^{\beta}(p)=0$ for all $p$ in $\beta \mathbb{N}-\mathbb{N}$, consequently $(h g)^{*}(p)=0$ for all $p$ in $\beta \mathbb{N}-\mathbb{N}$ and for all $g$ in $C^{*}(\mathbb{N})$. Since $\lim _{n \rightarrow \infty}\left(n^{s} e^{-n}\right)=0$ for each $s$ in $\mathbb{N}$, this clearly implies $(h g)^{*}(p)=0$ for all $p$ in $\beta \mathbb{N}-\mathbb{N}$ and for all $g$ in $A$. Hence $h$ belongs to every free maximal ideal in $A$, yet $h$ does not belong to $C_{K}(\mathbb{N})$. 


\section{On a question RAISED By Redlin AND Watson}

Redlin and Watson [5] raised the following question: Given a realcompact space $X$, does there exist in some sense a minimal algebra $A_{m}$ over $\mathbb{R}$ for which $X$ is $A_{m}$-compact? In this section we give an answer to this question. We recall the well-known fact that $X$ is $\sigma$-compact and locally compact if and only if $\beta X-X$ is a zero-set in $\beta X$ (see [6], Exercise 1B).

Consider any noncompact, $\sigma$-compact and locally compact space $X$. Then there exists an $f$ in $C^{*}(X)$ for which $\beta X-X=Z_{\beta X}\left(f^{\beta}\right)$. Let $g=f^{-1}$ and $A=$ $\mathcal{A}\left(C^{*}(X) \cup\{g\}\right)$. Then Theorem 3.2 implies that $X$ is $A$-compact. Also $X$ is not $C^{*}$-compact. It might be tempting to conjecture that $A$ is the smallest subalgebra of $C(X)$ with respect to the set inclusion relation for which $X$ becomes $A$-compact. That this is false for a suitable choice of $X$ is established in the following example.

Example 4.1. Consider an $f$ in $C^{*}(\mathbb{N})$ such that $f(n)>0$ for each $n \in \mathbb{N}$ and $\lim _{n \rightarrow \infty} f(n)=0$. Then $\beta \mathbb{N}-\mathbb{N}=Z\left(f^{\beta}\right)$. Let $g=f^{-1}$ which belongs to $C(\mathbb{N})$ and set $B=\mathcal{A}\left(C^{*}(\mathbb{N}) \cup\{g\}\right)$. Then by Theorem 3.2, $\mathbb{N}$ becomes $B$-compact. Now define $D=\mathcal{A}\left(C^{*}(\mathbb{N}) \cup\left\{\log _{e}(1+g)\right\}\right)$. We shall show that $\mathbb{N}$ is $D$-compact and $D \varsubsetneqq$

$B$. Since $\lim _{n \rightarrow \infty} f(n)=0, \lim _{n \rightarrow \infty} \frac{\log _{e}(1+g(n))}{g(n)}=0$. Consequently $\frac{\log _{e}(1+g)}{g} \in$ $C^{*}(\mathbb{N}) \subset B$. Hence $\log _{e}(1+g)$ is a member of $B$. Thus $D$ is contained in $B$. To show that the inclusion relation is proper, we shall show that $g$ belongs to $D-B$. If not, then $g$ must be a polynomial of the members of the set $C^{*}(\mathbb{N}) \cup\left\{\log _{e}(1+g)\right\}$. This means that $g$ is of the form $g=f_{0}\left(\log _{e}(1+g)\right)^{m}+f_{1}\left(\log _{e}(1+g)\right)^{m-1}+\cdots+f_{m}$, where $f_{0}, f_{1}, \ldots, f_{m} \in C^{*}(\mathbb{N}), m \in \mathbb{N}$. This implies that $\frac{g}{\left(\log _{e}(1+g)\right)^{n}} \in C^{*}(\mathbb{N})-$ a contradiction to the fact that $\lim _{n \rightarrow \infty} \frac{g(n)}{\left.\log _{e}(1+g)\right)^{n}}=\infty$.

The above example prompts us to frame the following:

Conjecture. There does not exist any minimal subalgebra $A$ of $C(\mathbb{N})$, in the usual inclusion sense, for which $\mathbb{N}$ becomes $A$-compact.

Nevertheless we give an affirmative answer to Redlin and Watson's question by defining an ordering among the elements of $\Sigma(X)$ in a suitable way, where $\Sigma(X)$ denotes the set of all subalgebras of $C(X)$ containing $C^{*}(X)$. For each $A$ in $\Sigma(X)$, let $\alpha_{A}$ be the smallest cardinal number of a subfamily $\mathcal{G}_{A}$ of $A-C^{*}(X)$ with the property $A=\mathcal{A}\left(C^{*}(X) \cup \mathcal{G}_{A}\right)$. For any two $A, B$ of $\Sigma(X)$ we define $A \prec B$ if and only if $\alpha_{A} \leq \alpha_{B}$. Then $\prec$ becomes a preorder on $\Sigma(X)$ with respect to which an arbitrary pair of members of $\Sigma(X)$ can be compared.

Theorem 4.2. Let $X$ be a realcompact space. Then there exists a minimal algebra $A_{m}$ in $\Sigma(X)$ with respect to the ordering $\prec$ for which $X$ becomes $A_{m}$-compact.

Lemma 4.3. Given $p \in \beta X$ and $A \in \Sigma(X), M_{A}^{p}$ is real if and only if $f^{*}(p)$ is a real number for each $f$ in $A$.

The proof of the lemma is quite similar to that of Theorem 8.4 of [2].

Proof of the theorem. The proof is trivial when $X$ is compact. So suppose that $X$ is not compact. Since $X$ is realcompact, in view of Remark 3.3 we have a subset $\mathcal{F}_{m}$ of $C^{*}(X)$ with a smallest cardinal number $\alpha$ with the property $\beta X-X=$ $\bigcup_{f \in \mathcal{F}_{m}} Z\left(f^{\beta}\right)$ and $Z\left(f^{\beta}\right) \neq \emptyset$ for each $f \in \mathcal{F}_{m}$. It is clear that each $f$ in $\mathcal{F}_{m}$ is a unit of $C(X)$ and moreover $f^{-1}$ belongs to $C(X)-C^{*}(X)$. Let $A_{m}=\mathcal{A}\left(C^{*}(X) \cup\left\{f^{-1}\right.\right.$ : 
$\left.\left.f \in \mathcal{F}_{m}\right\}\right)$. Then by Theorem 3.2, $X$ is $A_{m}$-compact, also $\alpha_{A_{m}} \leq \alpha$. Assume that for some $A$ in $\Sigma(X), X$ is $A$-compact. To complete the proof it is enough to show that $\alpha \leq \alpha_{A}$. Now there exists a subset $\mathcal{G}_{A}$ of $A-C^{*}(X)$ with cardinal number $\alpha_{A}$ such that $A=\mathcal{A}\left(C^{*}(X) \cup \mathcal{G}_{A}\right)$.

We claim that for each $p$ in $\beta X-X$, there exists a $g$ in $\mathcal{G}_{A}$ with $g^{*}(p)=\infty$. If not, then there exists a point $q$ in $\beta X-X$ such that for each $h$ in $\mathcal{G}_{A}, h^{*}(q)$ is real. Now since $X$ is $A$-compact and $M_{A}^{q}$ is hyperreal, by Lemma 4.3 , there exists a $g$ in $A$ for which $g^{*}(q)=\infty$. Since $g$ can be expressed as $g=t\left(g_{1}, g_{2}, \ldots, g_{n}\right)$, where $g_{1}, g_{2}, \ldots, g_{n}$ are members of $\mathcal{G}_{A}$ and $t$ is a polynomial in these $n$ variables with coefficients from $C^{*}(X)$, it follows that $g^{*}(q)$ is a real number - a contradiction. Let $\mathcal{F}_{A}=\left\{(g \vee \mathbf{1})^{-1}: g \in \mathcal{G}_{A}\right\}$; then each member of $\mathcal{F}_{A}$ is a positive real-valued bounded function on $X$, taking values arbitrarily near to zero. Therefore in view of the above observation one can write $\beta X-X=\bigcup\left\{Z\left(f^{\beta}\right): f \in \mathcal{F}_{A}\right\}$ with $Z\left(f^{\beta}\right) \neq \emptyset$ for each $f$ in $\mathcal{F}_{A}$. Hence by the definition of $\alpha$, it is less than or equal to the cardinal number of the family $\mathcal{F}_{A}$ and consequently $\alpha \leq \alpha_{A}$.

The authors wish to thank the referee for suggesting various improvements of the paper.

\section{REFERENCES}

[1] C. E. Aull, Rings of Continuous Functions, Dekker, New York, 1985. MR 86g:54001

[2] L. Gillman and M. Jerison, Rings of Continuous Functions, Springer-Verlag, New York, 1976. MR 53:11352

[3] C. W. Kohls, Ideals in Rings of Continuous Functions, Fund. Math., 45(1957), 28-50. MR 21:1517

[4] D. Plank, On a class of subalgebras of $C(X)$ with application to $\beta X-X$, Fund. Math., 64(1969), 41-54. MR 39:6266

[5] L. Redlin and S. Watson, Maximal ideals in subalgebras of $C(X)$, Proc. Amer. Math. Soc., 100(1987), 763-766. MR 88f:54031

[6] R. C. Walker, The Stone- Čech compactification, Springer- Verlag, Berlin, Heidelberg, New York, 1974. MR 52:1595

(S. K. Acharyya) Department of Pure Mathematics, University of Calcutta, 35, Ballygunge Circular Road, Calcutta 700019, India

(K. C. Chattopadhyay and D. P. Ghosh) Department of Mathematics, University of BURDWAN, BURdWAN 713104, INDIA 